\title{
Dificultades de lenguaje en niños preescolares con antecedente de prematuridad extrema
}

\author{
MARIANGELA MAGGIOLO L. ${ }^{1}$, VIRGINIA VARELA M. ${ }^{1,2}$, \\ CLAUDIA ARANCIBIA S. ${ }^{1}$, FELIPE RUIZ M. ${ }^{3}$ \\ 1. Escuela de Fonoaudiología, Facultad de Medicina, Universidad de Chile. \\ 2. Servicio de Pediatría, Centro de Referencia de Salud Cordillera Oriente. \\ 3. Clínica Psiquiátrica Universitaria, Universidad de Chile.
}

\begin{abstract}
Language difficulties in preschool children with a history of extreme prematurity

Introduction: Preterm infants are prone to present language development difficulties. There is evidence that verbal deficits are common and adversely affect social interaction as well as school learning. In Chile, these skills are not evaluated by the premature follow-up program; therefore, the extent of this problem is unknown. The objective of this study is to describe the language difficulties of a group of preschoolers with a history of extreme prematurity. Patients and Methods: Thirty children aged 4 and 5 years old, with a history of extreme prematurity, but without severe neurological damage or hearing loss were evaluated through language tests at the Premature Follow-up Polyclinic of the Eastern Cordillera Health Reference Center. Results: $73.3 \%$ of the children assessed had deficits in some area of the language. Of these, $77.3 \%$ had comprehensive and expressive difficulties. In this group, $86.4 \%$ showed significant difficulties in narrative skills. Discussion: A high preterm infant proportion presents language difficulties in preschool, resulting in the need of including specific intervention programs that promote better language development for this population.

(Key words: Prematurity, language, preschooler, learning).

Rev Chil Pediatr 2014; 85 (3): 319-327
\end{abstract}

\section{RESUMEN}

Introducción: Los niños prematuros son una población vulnerable a presentar dificultades en su desarrollo lingüístico posterior. Existe evidencia que los déficits verbales son frecuentes y que repercuten negativamente en la interacción social y en el aprendizaje escolar. En Chile, estas habilidades no son evaluadas por el programa de seguimiento de prematuros, desconociéndose la magnitud de este problema. El objetivo fue describir

Este trabajo fue financiado por el Proyecto de Investigación No 129-09, Escuela de Fonoaudiología, Facultad de Medicina, Universidad de Chile.

Recibido el 25 de junio de 2013, devuelto para corregir el 16 de octubre de 2013, segunda versión 10 de diciembre de 2013 , tercera versión 20 de enero de 2014, aceptado para publicación el 17 de abril de 2014.

Este trabajo cumple con los requisitos sobre consentimiento /asentimiento informado, comité de ética, financiamiento, estudios animales y sobre la ausencia de conflictos de intereses según corresponda.

Correspondencia a:

Prof. Mariangela Maggiolo L.

E-Mail: mmaggiol@med.uchile.cl 
las dificultades del lenguaje en un grupo de prematuros extremos en edad preescolar. Pacientes y Método: Treinta niños entre 4 y 5 años de edad, con antecedente de prematuridad extrema y sin hipoacusia ni daño neurológico severo, fueron evaluados con pruebas de lenguaje en el Policlínico de Seguimiento de Prematuros del Centro de Referencia de Salud Cordillera Oriente. Resultados: Un 73,3\% de los niños presentó déficit en algún área del lenguaje evaluada. De ellos, un $77,3 \%$ presentaron dificultades comprensivas y expresivas. En este grupo, un $86,4 \%$ evidenció dificultades en habilidades narrativas relevantes para el aprendizaje escolar. Discusión: Una alta proporción de niños prematuros presenta dificultades de lenguaje en la etapa preescolar, lo que hace necesario incluir programas de intervención específicos que promuevan un mejor desarrollo del lenguaje en esta población.

(Palabras clave: Prematuridad, lenguaje, preescolar, aprendizaje).

Rev Chil Pediatr 2014; 85 (3): 319-327

\section{Introducción}

Las cifras nacionales de prematuridad (recién nacidos $[\mathrm{RN}]$ con 36 o menos semanas de edad gestacional) se han estabilizado en los últimos años, representando cerca del 7,2\% del total de los nacimientos. De ellos, cerca del $1 \%$ corresponde a recién nacidos prematuros extremos (RNPE), menores de 32 semanas de gestación y/o de $1.500 \mathrm{~g}^{1,2}$. La sobrevida promedio de los RNPE, alcanza un $76 \%$, acercándose a un $90 \%$ en el grupo de los RN entre 1.250 y $1.500 \mathrm{~g}^{3,4}$.

Es un hecho conocido que los RNPE constituyen un grupo altamente vulnerable. Esta vulnerabilidad está determinada por la inmadurez de sus distintos sistemas, particularmente del sistema nervioso, el que está expuesto a estímulos y noxas ambientales para los cuales no está preparado. Así, un alto porcentaje de los niños que sobreviven presenta algún tipo de secuela en su neurodesarrollo ${ }^{5-8}$.

Las dificultades de los RNPE pueden manifestarse temprana o tardíamente ${ }^{5,9}$. Especial preocupación suscitan las dificultades más severas, tales como las alteraciones sensoriales (ceguera, sordera) y neuromotoras (parálisis cerebral $)^{6,10}$. Un estudio nacional en prematuros extremos de menos de $1.000 \mathrm{~g}$ evidenció que el $20 \%$ de ellos presenta un trastorno motor de moderado a severo y un $6 \%$ hipoacusia sensorio-neural bilateral moderada a severa ${ }^{11}$. Asimismo, numerosas investigaciones han demostrado secuelas a largo plazo, presentes en la edad preescolar y escolar, incluso en aque- llos niños considerados con inteligencia y estado neurológico normal ${ }^{8,9,12,13}$.

Existe evidencia que en los niños con antecedente de prematuridad, a partir de los 6 años de vida se manifiestan ciertos déficits en las funciones cognitivas superiores, difíciles de diagnosticar a temprana edad ${ }^{14}$. Así, pueden observarse trastornos en áreas específicas del desarrollo, tales como problemas de aprendizaje, déficit atencional con o sin hiperactividad, trastornos del lenguaje y conductuales. Estas dificultades impactan negativamente en la vida del niño, especialmente al ingreso a la escolaridad, por las exigencias académicas que ello implica ${ }^{13-18}$. Además, se han observado dificultades en la etapa escolar y adolescencia relacionadas con disfunciones en el rendimiento cognitivo general, funciones ejecutivas, memoria y aprendizaje verbal que podrían explicar sus problemas académicos. Tales déficits pueden incluso extenderse hasta la vida adulta ${ }^{7}, 19-22$.

Una de las funciones cognitivas esenciales que se afecta en los RNPE es el lenguaje. Algunos estudios evidencian que la prematuridad constituye un factor de riesgo para el desarrollo de una adecuada competencia lingüística ${ }^{22-24}$. Los trastornos del lenguaje se manifiestan como dificultad en el procesamiento fonológico, en la organización gramatical, el desarrollo semántico y las habilidades discursivas. Las dificultades en el discurso narrativo son particularmente relevantes, dada su relación con el aprendizaje escolar. Ello porque esta competencia permiten organizar las experiencias 
personales y los eventos que nos involucran, en una secuencia temporal con una estructura lingüística específica ${ }^{28-32}$.

En Chile hay pocos estudios relacionados al desempeño verbal de los niños prematuros, aún cuando existe una política nacional de seguimiento de esta población ${ }^{4}$. En ese contexto, los controles que se efectúan a estos niños se dirigen hacia su estado de salud general y al monitoreo de patologías incluidas en las Garantías Explícitas de Salud (GES) sobre la base de guías clínicas específicas ${ }^{1}$. Así, tienen acceso a evaluación audiológica e implementación de audífonos, para permitir un mejor desarrollo lingüístico, sólo en el caso de diagnóstico confirmado de hipoacusia. Sin embargo, no se incluye una evaluación regular de las habilidades verbales ni seguimiento de su progreso en todos los niños prematuros, aún cuando no presenten patologías asociadas a la génesis de problemas de lenguaje.

La importancia que conlleva un buen desarrollo del lenguaje para la vida escolar, social y familiar de los niños en las distintas etapas del desarrollo, pone de manifiesto la necesidad de contar con información acerca de las dificultades que los niños prematuros extremos puedan presentar en esta área y establecer su magnitud. De acuerdo a lo expuesto el propósito de este estudio es describir las dificultades de lenguaje que presenta un grupo de RNPE a la edad de 4 y 5 años.

\section{Pacientes y Método}

\section{Diseño del estudio}

Estudio de corte transversal descriptivo. El universo estuvo conformado por 48 niños nacidos prematuros con 32 o menos sem de gestación y/o menos de $1.500 \mathrm{~g}$ de peso al nacer, que asistían al Policlínico de Seguimiento de Prematuros Extremos del Centro de Referencia de Salud (CRS) Cordillera Oriente de Santiago de Chile. Todos pertenecían al estrato socioeconómico medio bajo. Criterios de inclusión: a) edad entre 4 y 5 años 11 meses; b) presentar niveles de atención, comprensión de instrucciones y condiciones de salud compatibles con la aplicación de instrumentos formales de evaluación del lenguaje. Criterios de exclusión: enfermedades neurológicas, sensoriales, congénitas, metabólicas o síndromes genéticos que impidieran la aplicación de los instrumentos escogidos para este estudio. Así, la muestra definitiva quedó constituida por 30 niños cuyo promedio de edad era de 4 años, 9 meses; $53 \%$ niñas. La mayoría de las madres de los niños de la muestra tenían más de 13 años de escolaridad (63,3\%). Un $63,3 \%$ de los niños recibían atención fonoaudiológica al momento de la evaluación, ya sea en el Policlínico de Seguimiento CRS o en establecimientos externos. Aquellos que asistían a terapia en el CRS $(\mathrm{n}=9)$ podían acceder a una sesión semanal individual durante tres meses, revaluándose su continuidad dependiendo de los avances por un igual número de sesiones. El propósito de este tratamiento es abordar las dificultades verbales específicas de cada uno de los niños. En el período de desarrollo del estudio, ninguno de ellos fue dado de alta. Los niños con atención fonoaudiológica en establecimientos externos (escuelas de lenguaje, $\mathrm{n}=10$ ) recibían una sesión a la semana de estimulación global de lenguaje de acuerdo a las normas de dichos establecimientos.

Este estudio fue aprobado por el Comité de Ética del CRS Cordillera Oriente. A los cuidadores de los niños se les solicitó firmar un consentimiento informado. La totalidad de los cuidadores accedió a participar.

\section{Instrumentos de evaluación}

Se midió el lenguaje de los niños en la Unidad de Fonoaudiología del CRS Cordillera Oriente, en una sala con las condiciones adecuadas para este fin. La aplicación de las pruebas fue efectuada por dos licenciados en Fonoaudiología de la Universidad de Chile, entrenados y supervisados por la fonoaudióloga titular del policlínico, quien además realizó el análisis e interpretación de los resultados obtenidos. La variable en estudio, problemas de lenguaje, se definió operacionalmente como un déficit en alguno de los niveles lingüísticos: fonológico, semántico, gramatical y del discurso. El nivel fonológico corresponde al manejo de los sonidos del lenguaje y a la forma cómo ellos se organizan en las palabras. El ni- 
vel semántico representa el contenido del lenguaje en cuanto a vocabulario y relaciones entre conceptos; el nivel gramatical da cuenta de la estructuración de las palabras en oraciones. Finalmente, el discurso es una unidad lingüística más compleja que implica organizar las oraciones de manera coherente, para cumplir funciones comunicativas (describir, argumentar, narrar, entre otras). Esta definición operacional de problemas de lenguaje no incluye las dificultades del habla (dislalias, tartamudez). Además se consideró el grado de compromiso de la comprensión para clasificar los déficits en mixtos (dificultades en comprensión y expresión lingüística) o expresivos (dificultades sólo de la expresión verbal).

Se aplicaron cuatro test para evaluar el lenguaje oral de amplio uso en el ámbito fonoaudiológico nacional. Todos poseen normas de referencia a población infantil chilena y cuentan con la validez y confiabilidad requeridas de acuerdo a los criterios psicométricos habituales. A continuación se describe brevemente cada uno de ellos.

\section{Test para Evaluar Procesos de Simplificación Fonológica revisado (TEPROSIF-r)}

Evalúa el nivel fonológico del lenguaje sobre la base de analizar la presencia de procesos fonológicos de simplificación en una lista de palabras estímulos. Su estandarización cuenta con normas para niños entre entre los 3 y los 6 años 11 meses. El nivel de confiabilidad alcanza a 0,90 y la validez es de $0,92{ }^{25}$.

\section{Test Exploratorio de Gramática Española de A. Toronto (TEGE)}

Este instrumento consta de una subprueba expresiva y otra comprensiva para evaluar tipos de oraciones, pronombres, verbos, adjetivos y preposiciones en niños entre 3 y 7 años 11 meses. Está estandarizado en Chile y cuenta con análisis de confiabilidad (subprueba expresiva: 0,77 y subprueba receptiva: 0,83 ). La puntuación obtenida en cada subprueba se interpreta según los percentiles propuestos por el instrumento. Los puntajes iguales o menores al percentil 10 corresponden a un déficit gramatical ${ }^{26}$.

\section{Test para la Comprensión Auditiva del}

Lenguaje de E. Carrow (TECAL)

Evalúa la comprensión del lenguaje en secciones de vocabulario, morfología y sintaxis en niños entre 3 y 6 años 11 meses. El índice de confiabilidad es de $0,77 \mathrm{y}$ el de validez de $0,80^{27}$.

\section{Método de Evaluación del Discurso Narrativo (EDNA)}

Posee dos subpruebas, una de producción y otra de comprensión de narraciones. Consiste en evaluar la capacidad para narrar y comprender historias. Se usan tres cuentos infantiles originales, balanceados en cuanto a longitud y complejidad discursivas. El método se aplica a niños entre 3 y 10 años 11 meses, cuenta con normas de referencia chilenas y valores de validez y confiabilidad de 0,66 y 0,84 respectivamente ${ }^{28}$.

El desempeño final de los niños incluidos en la muestra, se catalogó de acuerdo a dos categorías: normal o déficit. El rendimiento se consideró deficitario si al menos en una de las pruebas aplicadas el puntaje obtenido fue igual o inferior al percentil 10 o se ubicó en la segunda desviación estándar (DE) bajo la media de su rango etario, dependiendo de cada test.

\section{Análisis estadístico}

Los datos obtenidos fueron recopilados en una planilla Excel y analizados en términos descriptivos. En primer lugar se estableció la proporción de niños con problemas de lenguaje en la muestra; luego se determinó la distribución de ellos según la afectación en comprensión y expresión. Se comentan en general los niveles lingüísticos comprometidos.

\section{Resultados}

En primer término se presenta una descripción clínica individual de la muestra estudiada en relación a: edad gestacional, peso al nacer, Apgar, días de hospitalización y antecedentes clínicos (tabla 1).

El análisis del desempeño de lenguaje en la muestra reveló que $73,3 \%(\mathrm{n}=22)$ presentó 
Tabla 1. Características clínicas

\begin{tabular}{|c|c|c|c|c|c|c|}
\hline Sujeto & $\begin{array}{c}\text { Edad } \\
\text { gestacional } \\
\text { (semanas) }\end{array}$ & $\begin{array}{c}\text { Peso al } \\
\text { nacer } \\
\text { (g) }\end{array}$ & & & $\begin{array}{l}\text { Días } \\
\text { hospita- } \\
\text { lización }\end{array}$ & Antecedentes clínicos al momento del alta \\
\hline 1. & 31 & 1.445 & 7 & 8 & 35 & Signos de piramidalismo \\
\hline 2. & 30 & 1.475 & 8 & 8 & 30 & Membrana hialina tratada. EOA normales \\
\hline 3. & 31 & 1.448 & 8 & 9 & 30 & Síndrome hipotónico, signos de piramidalismo \\
\hline 4. & 28 & 1.165 & 8 & 9 & 57 & $\begin{array}{l}\text { Ant. familiares de alteraciones de lenguaje, HIV grado III, paraparesia } \\
\text { mínima, ventriculomegalia izquierda }\end{array}$ \\
\hline 5. & 30 & 970 & 8 & 8 & 88 & Síndrome hipertónico predominio izquierdo, LPV izquierda sin HIC \\
\hline 6. & 27 & 585 & 7 & 8 & 125 & HIC Izquierda, hemorragia pericerebelosa \\
\hline 7. & 26 & 885 & 4 & 8 & 88 & $\begin{array}{l}\text { Hipoplasia hemisferio-cerebeloso, ventriculomegalia bilateral leve } \\
\text { sin HIC }\end{array}$ \\
\hline 8. & 28 & 1.070 & 4 & 7 & 60 & Síndrome hipertónico, HIC LPV DBP oxígeno dependiente \\
\hline 9. & 31 & 1.405 & 5 & 8 & 60 & $\begin{array}{l}\text { comportamiento motor similar a Hemiparesia doble espática, } \\
\text { microcefalia, hiperlaxitud ligamentosa, }\end{array}$ \\
\hline 10. & 29 & 775 & 8 & 9 & 71 & DBP leve, sepsis por $S$. aureus \\
\hline 11. & 27 & 1.055 & 8 & 9 & 58 & EDPV \\
\hline 12. & 29 & 970 & 7 & 9 & 60 & Bronquitis, ictericia, apnea, ECN obstrucción intestinal operada \\
\hline 13. & 31 & 1.250 & 8 & 9 & 42 & EMH, eco encefálica normal \\
\hline 14. & 28 & 1.225 & 5 & 8 & 90 & LMPV izquierda, DBP, HIC grado 1 \\
\hline 15. & 28 & 920 & 8 & 9 & 55 & LPV I-II sin HIC DBP oxigenodependiente \\
\hline 16. & 27 & 1.080 & 9 & 9 & 55 & HIC III bilateral, EDPV \\
\hline 17. & 29 & 990 & 5 & 8 & 48 & $\begin{array}{l}\text { HIC III-IV derecha, infarto parietooccipital derecho, LPV, hemipare- } \\
\text { sia izquierda en evolución }\end{array}$ \\
\hline 18. & 28 & 1.225 & 8 & 9 & 49 & Ecoencefélica con EDPV ventricular \\
\hline 19. & 28 & 1.255 & 4 & 7 & 81 & $\begin{array}{l}\text { DBP oxigenodependiente, EDPV, catarata retina, sindrome pirami- } \\
\text { dal derecho }\end{array}$ \\
\hline 20. & 32 & 1.380 & 9 & 9 & 13 & EDPV, gemelo \\
\hline 21. & 26 & 890 & 8 & 8 & 82 & HIC I ventriculomegalia izquierda leve \\
\hline 22. & 29 & 1.485 & 2 & 8 & 76 & EMH, hipoglicemia DBP oxigenodependiente, \\
\hline 23. & 30 & 1.235 & 7 & 9 & 50 & Eco cerebral normal \\
\hline 24. & 26 & 735 & 8 & 8 & 89 & DBP oxigenodependiente HIC izquierda I \\
\hline 25. & 29 & 1.250 & 5 & 8 & 56 & DBP LPV Izquierda \\
\hline 26. & 32 & 1.490 & 8 & 9 & 40 & Sin antecedentes \\
\hline 27. & 29 & 1.315 & 8 & 9 & 36 & Sin antecedentes \\
\hline 28. & 32 & 1.360 & 8 & 9 & 40 & PEG EDPV \\
\hline 29. & 31 & 1.140 & 8 & 9 & 45 & EMH, osteopenia y retinopatía, gemela \\
\hline 30. & 31 & 1.225 & 8 & 9 & 45 & EMH Eco encefálica normales, gemela \\
\hline
\end{tabular}

déficit de lenguaje en uno o más de sus niveles, mientras que sólo un $26,7 \%$ tuvo un desempeño esperado para su edad.

En relación al grupo de niños que tiene comprometidas las habilidades lingüísticas, un $77,3 \%(\mathrm{n}=17)$ presentó un déficit mixto, es decir, tienen dificultades tanto en la expresión como en la comprensión del lenguaje. El $22,7 \%$ restante $(\mathrm{n}=5)$ sólo manifestó dificultades en la expresión verbal (figura 1). 


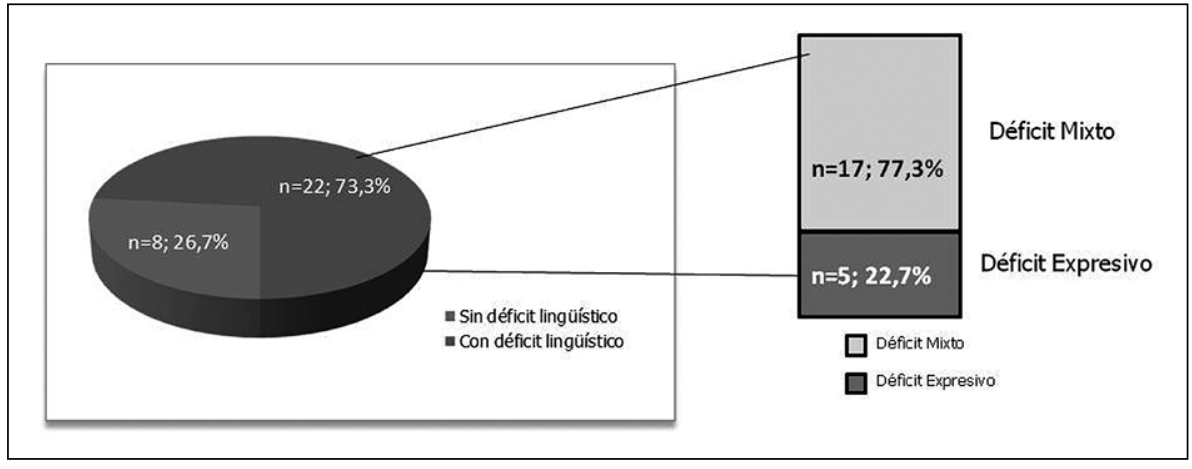

Figura 1. Proporción de compromiso de habilidades lingüísticas.



Figura 2. Distribución de la muestra según tipo de dificultades de lenguaje.
En relación al grupo con dificultades expresivas, los niños evidencian fundamentalmente problemas fonológicos y en la expresión narrativa. En el grupo de niños con déficit comprensivo-expresivo, los problemas son más variados y se combinan de modo diferente; $\sin$ embargo, en la mayor parte de ellos $(n=6)$ se observó la coexistencia de dificultades fonológicas, gramaticales y de discurso narrativo, así como la combinación de déficit gramaticales y de discurso narrativo $(\mathrm{n}=5)$ (figura 2$)$.

Cabe destacar que de la totalidad de los niños con déficit de lenguaje, ya sea expresivo o mixto, 19 presentaron problemas en las habi- lidades narrativas, lo que representa el $86,4 \%$ de la muestra. Este resultado es relevante dada la relación que existe entre esta habilidad y el rendimiento escolar.

\section{Discusión}

La heterogeneidad en cuanto a las áreas del desarrollo afectadas en los prematuros es amplia, sin embargo existe evidencia que algunas se alteran de modo especial, como el lenguaje. Los hallazgos de esta investigación son concordantes con la evidencia bibliográfica en lo 
que respecta al déficit en habilidades verbales que presentan los niños prematuros extremos, pues más del $70 \%$ de los niños evaluados muestra rendimientos disminuidos en algún aspecto lingüístico ${ }^{22-24}$. El análisis demuestra también que dos tercios de ellos tiene compromiso del lenguaje tanto comprensivo como expresivo, lo que refleja un grado de severidad mayor del déficit, pues se sabe que en el desarrollo normal las habilidades comprensivas preceden a las expresivas. Los niveles lingüísticos afectados corresponden principalmente a la fonología, gramática y discurso narrativo.

Un estudio reciente de seguimiento en niños prematuros australianos evaluados en la edad escolar refiere que el $38,1 \%$ de ellos presentó bajo desempeño en aspectos lingüísticos y matemáticos, reportando que pobres habilidades lectoras estaban asociadas a un bajo desempeño en vocabulario expresivo y conciencia fonológica ${ }^{29}$.

Otra investigación muestra que los problemas de lenguaje pueden aparecer en distintas etapas de la escolaridad en niños de pre término, comprometiendo diferentes habilidades, como procesamiento fonológico, vocabulario, comprensión verbal, memoria verbal, recuerdo de oraciones, comprensión de instrucciones, entre otras ${ }^{17}$. Hallazgos similares revela un estudio en niños prematuros fineses en edad preescolar, en el que se describe que a los 4 años manifiestan dificultades en comprensión lingüística, nominación rápida y discriminación auditiva ${ }^{30}$.

Un metaanálisis reciente, efectuado sobre 12 investigaciones relacionadas con las habilidades de lenguaje en niños de pretérmino y/o de muy bajo peso al nacer, evidencia que la competencia lingüística está reducida en este grupo de niños y que el déficit permanece en la edad escolar ${ }^{31}$. Se señala además que las investigaciones que dan cuenta del desempeño en otras habilidades verbales, como es el discurso, son escasas. En ese sentido, esta investigación aporta evidencia preliminar en cuanto al discurso narrativo en el grupo de niños estudiado, pues más de un $80 \%$ de ellos presenta déficit en este ámbito. La narración se manifiesta cuando los niños cuentan una experiencia personal en una conversación, otorgándole sentido y organizando sus experiencias a través de sus relatos; así, las dificultades narrativas implican que los niños tienen problemas para organizar sus experiencias y compartirlas con otros ${ }^{28}$. El discurso narrativo es más descontextualizado e implica el manejo de estructuras lingüísticas complejas, acercándose así a las características del lenguaje escrito, por lo que se considera un buen predictor del éxito escolar ${ }^{32}$. Al respecto, una investigación, efectuada en Australia en niños prematuros en edad escolar, refiere que estos tienen dificultades al formular sus narraciones en comparación con sus homólogos de término. Aún cuando no se observaron diferencias significativas entre estos grupos, los niños prematuros evidenciaron mayor número de latencias e interrupciones en sus relatos y por ende necesitaron mayor tiempo de formulación ${ }^{33}$.

Los resultados en el grupo estudiado constituyen una alerta en cuanto al futuro desempeño académico de los prematuros extremos, dado que el déficit del lenguaje y, en particular, del discurso, presentes en la edad preescolar, habitualmente se mantiene al ingreso a la escolaridad básica, si no se interviene oportuna y específicamente.

Una limitación del presente trabajo es no contar con un grupo control de niños nacidos de término con iguales características a las del grupo estudiado. Lo anterior, habría permitido determinar el riesgo relativo de la condición de prematuro extremo para desarrollar problemas de lenguaje, aspecto que debería ser incorporado en investigaciones posteriores. Sin embargo, en Chile existen antecedentes acerca de la magnitud de las dificultades de lenguaje en preescolares de término. Así, un estudio analizó el desempeño lingüístico en estos niños sin alteraciones sensoriales ni neurológicas y de nivel sociocultural bajo de la comuna de Recoleta. Se aplicaron las mismas pruebas de lenguaje que en el presente trabajo, con excepción del discurso narrativo. Los resultados indicaron que un $48,8 \%$ de los niños presentó problemas de lenguaje ${ }^{34}$. Al comparar esta evidencia con los resultados del presente trabajo podría sugerirse una condición de mayor vulnerabilidad de los niños prematuros extremos a presentar problemas de lenguaje en el curso 
de su desarrollo, puesto que la proporción de dificultades lingüísticas es muy superior a la evidenciada en el grupo de niños de término.

Otro aspecto a considerar en estudios posteriores es la asociación entre algunas variables relevantes y el desempeño lingüístico, como sería la edad gestacional, escolaridad de la madre, tiempo de hospitalización, patologías asociadas, intervención fonoaudiológica, entre otras. En este sentido, los antecedentes recabados de la muestra estudiada son variados. Así por ejemplo, la escolaridad de la madre, no parece en esta muestra relacionarse con el desempeño obtenido por los niños, pues de aquellos que tienen bajo desempeño, dos tercios $(n=15)$ tienen madres con escolaridad mayor a 13 años. Algo similar ocurre en el grupo de niños que tuvieron buen desempeño lingüístico $(n=5)$. Esto llama la atención, pues la escolaridad de la madre es un factor que se asocia fuertemente al desarrollo del lenguaje de los niños tanto de los que presentan problemas como aquellos con desarrollo típico ${ }^{24}$. La edad gestacional promedio $(29,1$ semanas para el grupo de niños con buen rendimiento y 29 semanas para el grupo de niños con problemas) tampoco difiere. Con respecto a los antecedentes clínicos, los niños con problemas de lenguaje evidencian características variadas que no sugieren, al menos en este análisis, una posible relación entre el grado de severidad del déficit de lenguaje de los niños (que a su vez es de compromiso variable) y el diagnóstico al momento del alta. Sin embargo, en este sentido, vale la pena destacar que de los 8 niños que tienen un desarrollo lingüístico típico, 7 no tenían antecedentes clínicos relevantes al momento del alta, lo que sugiere que podría haber una asociación entre la ausencia de patologías relevantes y el buen desempeño en las habilidades verbales evaluadas.

En relación a la atención fonoaudiológica que recibía el $63,3 \%$ de los niños cuando fueron evaluados sólo se consignó como antecedente de cada sujeto. En las fichas respectivas de los niños que recibían terapia en el CRS se pudo constatar que, en general, la asistencia a las sesiones era poco frecuente, había períodos de abandono con reincorporación posterior, bajo seguimiento de las indicaciones al hogar, entre otros. Esta evidencia sugiere que estos factores pueden influir negativamente en el desempeño de los niños. Estudios posteriores debieran comparar el desempeño verbal de grupos de niños en función de la regularidad con que asisten a la terapia. En el caso de los niños que asistían a escuelas de lenguaje no se obtuvieron antecedentes en cuanto a la asistencia y contenidos abordados en la estimulación, por lo que no es factible establecer algún impacto de esta intervención sobre su rendimiento lingüístico. En particular, estudios de asociación entre las variables comentadas y el nivel de desempeño verbal podrían aportar en la identificación de factores que tienen mayor impacto en el desarrollo lingüístico de los prematuros extremos y cómo influyen en la severidad de estos trastornos.

La relevancia de una adecuada competencia lingüística para el desarrollo cognitivo, socio-afectivo y los aprendizajes escolares está lo suficientemente respaldada. Por ello, los datos obtenidos en el presente trabajo corroboran la necesidad de incorporar en el programa de seguimiento del prematuro extremo evaluaciones periódicas de la comunicación y del lenguaje, así como estrategias terapéuticas destinadas a prevenir dificultades lingüísticas y minimizar el impacto de los déficits ya existentes en edades posteriores.

\section{Referencias}

1.- Departamento de Estadísticas e Información de Salud (DEIS): Ministerio de Salud, Gobierno de Chile. htpp:// www.deis.cl., última visita 03/06/12.

2.- Hübner ME, Nazer J, Juárez de León G: Estrategias para mejorar la sobrevida del prematuro extremo. Rev Chil Pediatr 2009; 80: 551-9.

3.- Morgues $M$, Reyes $C$ : Informe técnico. Recién nacidos con menos de 32 semanas de edad gestacional. Sistema Nacional de Servicios de Salud de Chile. Quinquenio 2000-2004. www.prematuros.cl, última visita 06-062012.

4.- Morgues M, Henríquez MT, Tohá D, et al: Sobrevida del niño menor de 1500 g. en Chile. Rev Chil Obstet Ginecol 2002; 67: 100-5.

5.- Sastre-Riba $S$ : Prematuridad: Análisis y seguimiento de las funciones ejecutivas. Rev Neurol 2009; 48: 113-8. 
6.- Crunelle D, Le Normand MT, Delfosse MJ: Langage oral et écrit chez des enfants prématurés: résultats à $71 \frac{1}{2}$ ans. Folia Phoniatr Logop 2003; 55: 115-27.

7.- Narberhaus A, Segarra D: Trastornos neuropsicológicos y del neurodesarrollo en el prematuro. Anales de psicología 2004; 20: 317-26.

8.- Salt A, Redshaw M: Neurodevelopmental follow-up after preterm birth: follow up after two years. Early Hum Dev 2006; 82: 185-97.

9.- Deforge H, André M, Hascoët JM, et al: Développement cognitif et performances attentionnelles de l'ancien prématuré « normal » à l'âge scolaire. Arch Pediatr 2006; 13: 1195-201.

10.- Wood N, Marlow N, Costeloe K, Gibson A, Wilkinson A: Neurologic and developmental disability after extremely preterm birth. N Engl J Med 2000; 343: 378-84.

11.- Salas R, Sanhueza L, Maggi L: Factores de riesgo y seguimiento clínico en prematuros menores de $1000 \mathrm{~g}$. Rev Chil Pediatr 2006; 77: 577-88.

12.- Taylor G, Klein N, Minich N, Hack M: Verbal memory deficits in children with less than $750 \mathrm{~g}$ birth weight. Child Neuropsychol 2000; 6: 49-63.

13.- Taylor G, Hack M, Klein N: Attention deficits in children with $<750$ gm birth weight. Child Neuropsichol 1998; 4: 21-34.

14.- Casasbuenas O.L: Seguimiento neurológico del recién nacido pretérmino. Rev Neurol 2005; 40: 65-7.

15.- Rickards A, Kitchen W, Doyle L, et al: Cognition, school performance, and behaviour in very low birth weight and normal birth weight children at 8 years of age: a longitudinal study. J Dev Behav Pediatr 1993; 14: 363 8.

16.- Peña M, Pittaluga E, Farkas $C H$ : Adquisición fonológica en prematuros. Rev Neurol 2010; 50: 12-8.

17.- Anderson P, Doyle L: Cognitive and educational deficits in children born extremely preterm. Semin Perinatol 2008; 32: 51-8.

18.- Marlow $N$ : Outcome following extremely preterm birth. Women's Health Medicine 2006; 16: 141-6.

19.- Narberhaus A, Segarra-Castells MD, Pueyo-Benito R, Botet-Mussons F, Junqué C: Disfunciones cognitivas a largo plazo en sujetos prematuros con hemorragia intraventricular. Rev Neurol 2008; 47: 57-60.

20.- Anderson P, Doyle L: Executive functioning in schoolaged children who were born very preterm or with extremely low weight birth in the 1990s. Pediatrics 2004; 114: 50-7.

21.- Hack M, Flannery D, Schluchter M, Cartar L, Borawski $E$, Klein $N$ : Outcomes in young adulthood for very low birth weight infants. N Engl J Med 2002; 346: 149-57.

22.- Ishii C, Shizue C, Isotani S, Perissinoto J: Caracterização de comportamentos lingüisticos de crianças nascidas prematuras aos quatro anos de idade. Rev CEFAC 2006; 8: 147-54.

23.- Rizzotto C, Wetters M, Lahorgue M: Clinical assessment of languaje development in children at age 3 years that were born pretem. Arq Neuropsiquiatr 2006; 64: 92631.

24.- Sansavini A, Guarini A, Alessandroni R, Faldella G, Giovanelli $G$, Salvioli $G$ : Are early grammatical and phonological working memory abilities affected by preterm birth?. J Commun Disord 2007; 40: 239-56.

25.- Pavez MM, Maggiolo M, Coloma C: Test para evaluar procesos de simplificación fonológica. Versión revisada. TEPROSIF-r, Santiago de Chile: Ediciones Pontificia Universidad Católica de Chile, 2008.

26.- Pavez MM: Test exploratorio de gramática española de A. Toronto. TEGE, Santiago de Chile: Ediciones Pontificia Universidad Católica de Chile, 2002.

27.- Pavez MM: Test de evaluación de la comprensión auditiva del lenguaje. TECAL, Santiago de Chile: Editorial Universitaria, 2006.

28.- Pavez M, Coloma C, Maggiolo M: El desarrollo narrativo en niños. Una propuesta práctica para la evaluación y la intervención en niños con trastorno del lenguaje. Ars Medica, 2008.

29.- Wocadlo C, Rieger I: Phonology, rapid naming and academic achievement in very preterm children at eight years of age. Early Hum Dev 2007; 83: 367-77.

30.- Jansson-Verkasalo E, Valkama M, Vainionpää $L$, Pääkkö E, IIkko E, Lehtihalmes M: Language development in very low birth weight preterm children: A follow-up study. Folia Phoniatr Logop 2004; 56: 10819.

31.- Barre N, Morgan A, Doyle L, Anderson P: Language abilities in children who were very preterm and/or very low birth weight: A meta-analysis. The Journal of Pediatrics 2011; 158: 766-74.

32.- Bishop D, Edmundson A. Language impaired at 4- years old: distinguishing transient from persistent impairment. $\mathrm{J}$ of Speech and Hearing Disord 1987; 52 156-73.

33.- Crosbie S, Holm A, Wandschneider S, Hemsley G. Narrative skills of children born preterm. Int J Lang Commun Disord 2011; 46: 83-94.

34.- Schonhaut L, Maggiolo M, De Barbieri Z, Rojas P, Salgado A. Dificultades del lenguaje en preescolares: concordancia entre el test TEPSI y la evaluación fonoaudiológica. Rev Chil Pediatr 2007; 78: 369-75. 